\title{
Sectional and Ricci Curvature for Three-Dimensional Lie Groups
}

\author{
Gerard Thompson ${ }^{1}$ and Giriraj Bhattarai ${ }^{2}$ \\ ${ }^{1}$ Department of Mathematics, The University of Toledo, Toledo, $\mathrm{OH} 43606$, USA \\ ${ }^{2}$ Bristol Community College, Fall River, MA 02720, USA \\ Correspondence should be addressed to Gerard Thompson; gerard.thompson@utoledo.edu
}

Received 12 July 2016; Accepted 31 October 2016

Academic Editor: K. F. C. Yiu

Copyright (C) 2016 G. Thompson and G. Bhattarai. This is an open access article distributed under the Creative Commons Attribution License, which permits unrestricted use, distribution, and reproduction in any medium, provided the original work is properly cited.

Formulas for the Riemann and Ricci curvature tensors of an invariant metric on a Lie group are determined. The results are applied to a systematic study of the curvature properties of invariant metrics on three-dimensional Lie groups. In each case the metric is reduced by using the automorphism group of the associated Lie algebra. In particular, the maximum and minimum values of the sectional curvature function are determined.

\section{Introduction}

In [1] Milnor investigated curvature properties of invariant metrics on Lie groups particularly in dimension three. A much more recent paper [2] studied the solution of Killing's equations for invariant metrics in dimensions two and three. In certain special cases it was found that some of the metrics corresponded to spaces of constant curvature. In the present paper we reconsider metrics in dimension three and undertake an exhaustive study of sectional and Ricci curvature. Although Milnor used left-invariant metrics we prefer to use right-invariant metrics although of course it is not essential to do so.

In Section 2 we derive formulas in indices for the Riemann and Ricci tensors and scalar curvature of an invariant metric. In Sections 3 and 4 we study in detail curvature properties of invariant metrics in dimensions two and three corresponding to all possible nonabelian Lie algebra. However, in this paper we propose a new technique; that is, we shall reduce the metric using the automorphism group of the algebra. As a result the reduced metric depends only on one or two parameters and it makes it possible to obtain very explicit formulas for the Ricci tensor and sectional curvature function. In each case we find the maximum and minimum values of the sectional curvature function. Since the metrics have been reduced in an equivariant manner, the curvature properties that we find are definitive.
As regards notation, in Section 2 we use the summation convention on repeated indices until it becomes impractical to do so. In Section 4 corresponding to three-dimensional Lie algebra given in [3], we give a matrix Lie group $S$ whose Lie algebra gives the Lie algebra in question as well as the set of left and right-invariant vector fields and one-forms. Furthermore, we specify the Lie algebra of derivations by a $3 \times$ 3 matrix with Greek entries. The automorphism group, or at least its identity component, can be found by exponentiating the space of derivations. We shall also find it convenient to use an overall scaling of the metric: it is not absolutely necessary to do so and doing so does not change the curvature tensor nor the signs of the Ricci eigenvalues.

In terms of finding sectional curvature we shall compute it first of all on a collection of planes that are transverse to a given axis (generic case) and then on the residual set of planes that contain the given axis (nongeneric case). At the risk of some confusion, we parametrize the set of two-planes in both cases using the coordinates $(x, y)$.

\section{Formulas for Curvature and Ricci Tensors}

In this section we derive a formula for the Ricci tensor of a left or right-invariant metric $g_{i j}$ on a Lie group $G$. It is convenient to keep the metric visible since we cannot assume that we are working in an orthonormal frame. Thus, if $g_{i j}$ is right-invariant and $\left\{e_{i}\right\}$ is a basis for the right-invariant vector 
fields, $g_{i j}$ is a constant matrix. The compatibility of $g_{i j}$ with its Levi-Civita connection $\Gamma_{j k}^{i}$ gives

$$
g_{l j} \Gamma_{k i}^{l}+g_{i l} \Gamma_{k j}^{l}=0
$$

whereas the fact that the connection is torsion-free gives

$$
\Gamma_{k i}^{l}-\Gamma_{i k}^{l}=C_{k i}^{l} \text {. }
$$

From $\nabla_{e_{k}} e_{i}-\nabla_{e_{i}} e_{k}=\left[e_{k}, e_{i}\right]$, we have $\Gamma_{k i}^{l} e_{l}-\Gamma_{i k}^{l} e_{l}=C_{k i}^{l} e_{l}$. Now cycle the indices in (1) and use (2) to obtain

$$
\Gamma_{i j}^{m}=\frac{1}{2} g^{k m}\left(g_{i l} C_{k j}^{l}+g_{j l} C_{k i}^{l}+g_{k l} C_{i j}^{l}\right)
$$

Notice that in (3) the first two terms are symmetric in $i$ and $j$ and that the third term is skew-symmetric in $i$ and $j$. Now we find that the curvature is given by

$$
R_{l j k}^{i}=\Gamma_{j l}^{m} \Gamma_{k m}^{i}-\Gamma_{k l}^{m} \Gamma_{j m}^{i}+\Gamma_{m l}^{i} C_{j k}^{m}
$$

Notice that if we write (4) invariantly we get the usual formula for curvature: that is,

$$
R(X, Y) Z=\nabla_{Y} \nabla_{X}-\nabla_{X} \nabla_{Y} Z+\nabla_{[X, Y]},
$$

where in the last term we have the Lie algebra bracket. After a very long calculation (4) gives for the Ricci tensor

$$
\begin{aligned}
4 R_{i j}= & 2 g^{k p} g_{m q} C_{j k}^{m} C_{p i}^{q}-g^{k q} g^{m n} g_{i p} g_{j r} C_{n k}^{p} C_{q m}^{r} \\
& -2 C_{m k}^{k} g^{m p}\left(g_{j q} C_{p i}^{q}+g_{i q} C_{p j}^{q}\right)+2 C_{j k}^{m} C_{m i}^{k} .
\end{aligned}
$$

We note that $R_{i j}$ is symmetric as it must be for a metric connection and also that the fourth term is the Killing form up to a factor. Moreover, the third term vanishes for unimodular Lie algebra and the third and fourth terms vanish for nilpotent Lie algebra.

If we assume that we are in an orthonormal frame so that $g_{i j}=\delta_{i j}$, where $\delta_{i j}$ denotes the Kronecker delta, then we find after very long calculations that the curvature and Ricci tensors and scalar curvature are given by

$$
\begin{aligned}
& 4 R_{l j k}^{i} \\
& =\sum_{m} C_{j k}^{m}\left(C_{i m}^{l}-C_{l m}^{i}\right)+C_{i l}^{m}\left(C_{j m}^{k}-C_{k m}^{j}\right)+C_{j l}^{m} C_{i k}^{m} \\
& -C_{k l}^{m} C_{i j}^{m}+2 C_{j k}^{m} C_{i l}^{m}+\left(C_{m l}^{j}+C_{m j}^{l}\right)\left(C_{i m}^{k}+C_{k m}^{i}\right) \\
& -\left(C_{m l}^{k}+C_{m k}^{l}\right)\left(C_{i m}^{j}+C_{j m}^{i}\right), \\
& 4 R_{l j} \\
& =\sum_{k, m} 2\left(C_{m l}^{j}+C_{m j}^{l}\right) C_{k m}^{k}+2 C_{j k}^{m} C_{k l}^{m}-2 C_{j k}^{m} C_{l m}^{k} \\
& +C_{k m}^{j} C_{k m}^{l}
\end{aligned}
$$

$4 R$

$$
=-\left(4 \sum_{m}\left(\sum_{j} C_{m j}^{j}\right)^{2}+\sum_{j, k, m}\left(\left(C_{m j}^{k}\right)^{2}+2 C_{j k}^{m} C_{j m}^{k}\right)\right) .
$$

\section{Two-Dimensional Lie Group}

3.1. Dimension 2 Nonabelian. Nonzero brackets: $\left[e_{1}, e_{2}\right]=e_{2}$.

$$
S=\left[\begin{array}{ll}
e^{x} & y \\
0 & 1
\end{array}\right]
$$

Left-invariant vector fields: $D_{x}, e^{x} D_{y}$.

Left-invariant one-forms: $d x, e^{-x} d y$.

Right-invariant vector fields: $-\left(D_{x}+y D_{y}\right), D_{y}$.

Right-invariant one-forms: $-d x, d y-y d x$.

The only derivations are inner and the automorphism group coincides with the adjoint representation. As such and including an overall scaling the matrix of the metric may be reduced to the identity. The metric is of constant Gaussian curvature -1 .

\section{Three-Dimensional Lie Groups}

4.1. Algebra 3.1 (Heisenberg: Nilpotent). Nonzero brackets: $\left[e_{2}, e_{3}\right]=e_{1}$.

$$
S=\left[\begin{array}{lll}
1 & x & z \\
0 & 1 & y \\
0 & 0 & 1
\end{array}\right]
$$

Left-invariant vector fields: $D_{z}, D_{y}-x D_{z}, D_{x}$.

Left-invariant one-forms: $d z-x d y, d y, d x$.

Right-invariant vector fields: $D_{z}, D_{x}+y D_{z}, D_{y}$.

Right-invariant one-forms: $d z-y d x, d x, d y$.

The space of derivations is given by $\left[\begin{array}{ccc}\alpha+\delta & \epsilon & \phi \\ 0 & \alpha & \beta \\ 0 & \gamma & \delta\end{array}\right]$ and the group of automorphisms by $P=\left[\begin{array}{cc}\operatorname{det}(Q) & x^{t} \\ 0 & Q\end{array}\right]$, where $Q \in$ $G L(2, \mathbb{R})$ and $x \in \mathbb{R}^{2}$ is a column vector.

Consider a symmetric matrix $g$ given by $g=\left[\begin{array}{ll}a & b^{t} \\ b & C\end{array}\right]$. The action of the automorphism group on $g$ is given by

$$
\left[\begin{array}{cc}
a \operatorname{det} Q^{2} & \operatorname{det} Q\left(a x^{t}+b^{t} Q\right) \\
\operatorname{det} Q\left(a x+Q^{t} b\right) & a x x^{t}+x b^{t} Q+Q^{t} b x^{t}+Q^{t} C Q
\end{array}\right] .
$$

Since the metric is assumed to be Riemannian, $a>0$. As such we may define $x:=-(1 / a) b$ which has the effect of removing $b$ in $g$. Now we continue to normalize $g$ assuming that $b=0$ and $x=0$. As such $g$ may be reduced to $\left[\begin{array}{ll}a & 0 \\ 0 & I\end{array}\right]$, where $I$ is the $2 \times 2$ identity matrix. We find it convenient to replace $a$ by $2 a$ below so that the metric can be reduced to $\left[\begin{array}{ccc}2 a & 0 & 0 \\ 0 & 1 & 0 \\ 0 & 0 & 1\end{array}\right]$

4.1.1. Sectional Curvature. We shall now investigate the sectional curvature in detail: we wish to find the maximum and minimum values. In the group matrix $S$ above, group coordinates are denoted by $(z, x, y)$. Now, at the risk of confusion, we are going to use $(x, y)$ in a different sense: that is, as 
coordinates on the space of two-planes in the tangent space at the identity. Note that at the identity the invariant vector fields reduce simply to $\left\{D_{z}, D_{x}, D_{y}\right\}$, which play the role simply of "unit vectors" pointing along the three coordinate axes. As such almost every two-plane has a basis of the form $X=$ $x D_{z}+D_{x}, Y=y D_{z}+D_{y}$ for some $x, y \in \mathbb{R}$. Our task now is to compute $K(X, Y)=g(X, R(Y, X) Y) /(g(X, X) g(Y, Y)-$ $\left.g(X, Y)^{2}\right)$. Using the definitions we find that

$$
\begin{aligned}
R(Y, X) Y= & R\left(D_{y}+y D_{z}, D_{x}+x D_{z}\right)\left(D_{y}+y D_{z}\right) \\
= & R\left(D_{y}, D_{x}\right)\left(D_{y}+y D_{z}\right) \\
& +x R\left(D_{y}, D_{z}\right)\left(D_{y}+y D_{z}\right) \\
& +y R\left(D_{z}, D_{x}\right)\left(D_{y}+y D_{z}\right) .
\end{aligned}
$$

So

$$
K(x, y)=g(X, R(Y, X) Y)=\frac{2 a^{2} x^{2}+2 a^{2} y^{2}-3 a}{4 a\left(x^{2}+y^{2}\right)+2} .
$$

The partial derivatives are given by

$$
\begin{aligned}
& K_{x}=\frac{8 a^{2} x}{\left(1+2 a y^{2}+2 a x^{2}\right)^{2}}, \\
& K_{y}=\frac{8 a^{2} y}{\left(1+2 a y^{2}+2 a x^{2}\right)^{2}} .
\end{aligned}
$$

Clearly $(x, y)=(0,0)$ is the unique critical point. The Hessian of $K$ at $(0,0)$ is $64 a^{4}>0$ and $K_{x x}(0,0)=8 a^{2}>0$ so the origin yields a local and in fact global minimum of $K(0,0)=-3 a / 2$.

The only planes that have been omitted in the $(x, y)$ parametrization are those planes that contain the $z$-axis. As such, a basis may be chosen of the form $\left\{X=x D_{x}+y D_{y}, Y=\right.$ $\left.D_{z}\right\}$, where $x$ and $y$ are not both zero and we find that

$$
k(x, y)=g(X, R(Y, X) Y) \equiv \frac{a}{2} .
$$

We use $k$ rather $K$ to distinguish the nongeneric from the generic sectional curvature. We shall adhere to the same notation in the remaining cases below. Hence, the sectional curvature varies between $a / 2$ and $-3 a / 2$ inclusive.

4.1.2. Ricci Curvature. The Ricci tensor as a type $(1,1)$ tensor is $\left[\begin{array}{ccc}a & 0 & 0 \\ 0 & -a & 0 \\ 0 & 0 & -a\end{array}\right]$.

4.2. Algebra 3.2. Nonzero brackets: $\left[e_{1}, e_{3}\right]=e_{1},\left[e_{2}, e_{3}\right]=$ $e_{1}+e_{2}$.

$$
S=\left[\begin{array}{ccc}
e^{z} & z e^{z} & x \\
0 & e^{z} & y \\
0 & 0 & 1
\end{array}\right]
$$

Left-invariant vector fields: $e^{z} D_{x}, e^{z}\left(D_{y}+z D_{x}\right),-D_{z}$. Left-invariant one-forms: $e^{-z}(d x-z d y), e^{-z} d y, d z$.

Right-invariant vector fields: $D_{x}, D_{y}, D_{z}+(x+y) D_{x}+$ $y D_{y}$.

Right-invariant one-forms: $d x-(x+y) d z, d y-$ $y d z, d z$.

The space of derivations is given by $\left[\begin{array}{lll}\alpha & \beta & \gamma \\ 0 & \alpha & \delta \\ 0 & 0 & 0\end{array}\right]$.

4.2.1. Sectional Curvature. The metric can be reduced to $\left[\begin{array}{ccc}2 a & 0 & 0 \\ 0 & 1 & 0 \\ 0 & 0 & 1\end{array}\right]$, where $a>0$. Indeed the metric can first of all be diagonalized using the parameters $\beta, \gamma, \delta$ in the space of derivations introduced above. After diagonalization we have only one parameter left at our disposal but we choose also to use an overall scaling by a nonzero factor $C$ of the metric so as to make it as simple as possible. It is not essential to do so but it does help to simplify some of the formulas. The Riemann and Ricci curvature tensors are unaffected by such a scaling but the scalar, sectional curvatures, and Ricci eigenvalues are multiplied by $1 / C$.

Proceeding as we did for Algebra 3.1, almost all tangent planes at the origin have a basis of the form $\left\{X=D_{x}+\right.$ $\left.x D_{z}, Y=D_{y}+y D_{z}\right\}$. As such

$$
\begin{aligned}
R(Y, X) Y= & R\left(D_{y}+y D_{z}, D_{x}+x D_{z}\right)\left(D_{y}+y D_{z}\right) \\
= & R\left(D_{y}, D_{x}\right)\left(D_{y}+y D_{z}\right) \\
& +x R\left(D_{y}, D_{z}\right)\left(D_{y}+y D_{z}\right) \\
& +y R\left(D_{z}, D_{x}\right)\left(D_{y}+\beta D_{z}\right) .
\end{aligned}
$$

Hence,

$$
\begin{aligned}
& K(x, y) \\
& =\frac{(3 a+2) x^{2}-8 a x y+\left(4 a-2 a^{2}\right) y^{2}+4 a-2 a^{2}}{2\left(x^{2}+2 a y^{2}+2 a\right)} .
\end{aligned}
$$

The partial derivatives are given by

$$
\begin{aligned}
& K_{x}=\frac{-4 a\left(-2 a y-2 a y^{3}+y x^{2}+2 x a+2 x a y^{2}\right)}{4\left(2 a+2 a y^{2}+x^{2}\right)^{2}}, \\
& K_{y}=\frac{4 a x\left(2 a-2 a y^{2}+x^{2}+2 a x y\right)}{4\left(2 a+2 a y^{2}+x^{2}\right)^{2}} .
\end{aligned}
$$

Multiplying (18) by $x$ and adding $y$ times (19) gives $8 a x^{2}(2 y-x)=0$. Now $a>0$ so either $x=0$ or $x=2 y$. If $x=0$ (18) gives $2 a y\left(y^{2}+1\right)$ so we have $x=y=0$. If $x=2 y$ then (19) gives $a+a y^{2}+2 y^{2}=0$, which is impossible since $a>0$. Hence, the origin is the only critical point. The Hessian at the origin turns out to be -4 so that the origin gives a saddle point. 
This time the exceptional values of the sectional curvature are given by

$$
k=-\frac{\left(4 a-2 a^{2}\right) x^{2}+8 a x y+(3 a+2) y^{2}}{2\left(2 a x^{2}+y^{2}\right)} .
$$

To find the critical points we have to solve

$$
\begin{aligned}
& 4 a y\left(2 a x y+2 a x^{2}-y^{2}\right)=0 \\
& 4 a x\left(2 a x y+2 a x^{2}-y^{2}\right)=0
\end{aligned}
$$

The critical points are given by $y=\left(a \pm \sqrt{a^{2}+2 a}\right) x$ for which $k$ assumes the values $-(a / 2+1) \pm \sqrt{a^{2}+2 a}$. Hence, the maximum and minimum values are, respectively, $-(a / 2+1)+$ $\sqrt{a^{2}+2 a}$ and $-(a / 2+1)-\sqrt{a^{2}+2 a}$. Note that $a^{2}+2 a-(a / 2+$ $1)^{2}=(3 / 4)(a+2)(a-2 / 3)$. Hence, if $a<2 / 3$ the values of the sectional curvature will always be negative.

4.2.2. Ricci Curvature. The Ricci tensor as type $(1,1)$ is $\left[\begin{array}{ccc}a-2 & -1 & 0 \\ -2 a & -(a+2) & 0 \\ 0 & 0 & -(a+2)\end{array}\right]$. The eigenvalues are $-(a+2),-2 \pm \sqrt{a^{2}+2 a}$. Two eigenvalues are unconditionally negative and the third is negative, zero, or positive according as $a$ is less than, equal to, or greater than $\sqrt{5}-1$.

4.3. Algebra $3.3(a=1), 3.4(a=-1), 3.5(0<|a|<1)$. Nonzero brackets: $\left[e_{1}, e_{3}\right]=e_{1},\left[e_{2}, e_{3}\right]=a e_{2}$.

$$
S=\left[\begin{array}{ccc}
e^{z} & 0 & x \\
0 & e^{a z} & y \\
0 & 0 & 1
\end{array}\right]
$$

Left-invariant vector fields: $e^{z} D_{x}, e^{a z} D_{y}, D_{z}$.

Left-invariant one-forms: $e^{-z} d x, e^{-a z} d y, d z$.

Right-invariant vector fields: $D_{x}, D_{y}, D_{z}+x D_{x}+$ ayD .

Right-invariant one-forms: $d x-x d z, d y-a y d z, d z$.

If $a=1$ the space of derivations is given by $\left[\begin{array}{lll}\alpha & \beta & \gamma \\ \delta & \epsilon & \phi \\ 0 & 0 & 0\end{array}\right]$. The case $a=1$ is very special. It is the three-dimensional case of the algebra $\mathfrak{g}$ discussed in [1] which has the property that, for all $x, y \in \mathfrak{g},[x, y]=\lambda x+\mu y$ for some $\lambda, \mu \in \mathbb{R}$. It may be shown that in dimension $n$ this algebra is unique up to isomorphism and has nonzero brackets $\left[e_{i}, e_{n}\right]=e_{i}$ for $1 \leq$ $i \leq n-1$. Milnor shows that for such an algebra any leftinvariant metric has constant curvature -1 and in fact that these algebras are the only ones to have this property [1].

From our point of view we can explain the significance of this metric as follows. The space of derivations is given by the algebra of $n \times n$ matrices whose bottom row is zero but is otherwise arbitrary, which is of dimension $n(n-1)$ being in fact the Lie algebra of the affine group of $\mathbb{R}^{n-1}$. Of all algebra of dimension $n$ it has the largest dimensional automorphism group apart from the one-dimensional extension of it obtained by adding multiples of the identity and the abelian algebra $\mathbb{R}^{n}$. Having such a large automorphism group, it is possible to reduce the matrix of a left or right-invariant metric to the identity matrix. Thus, there is essentially only one invariant metric allowing for symmetry.

The remainder of this subsection is devoted to the case $(0<|a|<1, a=-1)$ for which the space of derivations is given by $\left[\begin{array}{lll}\alpha & 0 & \beta \\ 0 & \gamma & \delta \\ 0 & 0 & 0\end{array}\right]$. The metric can be reduced to $\left[\begin{array}{lll}1 & b & 0 \\ b & 1 & 0 \\ 0 & 0 & 1\end{array}\right]$, where $|b|<1$ and again we have used scaling to simplify the metric.

4.3.1. Sectional Curvature. The generic sectional curvature function $K(x, y)$ is given by

$$
\begin{aligned}
K & (x, y) \\
& =\frac{1}{4\left(1+x^{2}+y^{2}-b^{2}-2 b x y\right)\left(1-b^{2}\right)}\left(a^{2} b^{2}-4 a\right. \\
& +6 a b^{2}+b^{2}-2 a b^{4}-b^{4}-a^{2} b^{4} \\
& -\left(4 a^{2}+2 a b^{2}-b^{2}-5 a^{2} b^{2}\right) x^{2} \\
& \left.-\left(2 a b^{2}-5 b^{2}-a^{2} b^{2}+4\right) y^{2}\right) .
\end{aligned}
$$

To find the critical points we have to solve

$$
\begin{aligned}
& (1-a)\left(a b^{2} x+y a b x^{2}-a x-a x y^{2}+y^{3} b-y^{2} x\right) \\
& \quad=0 \\
& (1-a)\left(a b x^{3}-a x^{2} y+b^{2} y+b x y^{2}-y-x^{2} y\right)=0 .
\end{aligned}
$$

Multiplying (24) by $x$ and subtracting $y$ times (25) give

$$
y^{2}=a x^{2}
$$

Using (26) in (24) gives $(a+1 \pm 2 b \sqrt{a}) x^{2}+1-b^{2}=$ 0 . However, since $0 \leq|b|<1$ then $a+1 \pm 2 b \sqrt{a}>$ $(\sqrt{a}-1)^{2}>0$ and hence the origin is the only critical point. The Hessian at the origin turns out to be $-4 a(1-$ $a)^{2} /(1-b)^{2}(1+b)^{2}$ so that if $a>0$ the origin gives a saddle point and if $a<0$ a local extremum. We find also that $K_{x x}(0,0)=2 a(1-a) /\left(1-b^{2}\right)$ so that this local extremum is actually a local maximum given by $K(0,0)=$ $\left(b^{2}-4 a+6 a b^{2}+a^{2} b^{2}-b^{4}-2 a b^{4}-a^{2} b^{4}\right) / 4\left(1-b^{2}\right)^{2}$.

In the exceptional case we find that

$$
k(x, y)=\frac{\left(5 b^{2}-4-2 a b^{2}+b^{2} a^{2}\right) x^{2}+\left(2 a^{2} b^{3}+4 a b^{3}+2 b^{3}-8 a b\right) x y+\left(5 a^{2} b^{2}+b^{2}-2 a b^{2}-4 a^{2}\right) y^{2}}{4\left(1-b^{2}\right)\left(x^{2}+2 b x y+y^{2}\right)} .
$$


The partial derivatives are given by

$$
\begin{aligned}
& k_{x}=\frac{(2(a-1))\left(b x^{2}+(a+1) x y+a b y^{2}\right) y}{\left(x^{2}+2 b y x+y^{2}\right)^{2}}, \\
& k_{y}=-\frac{(2(a-1))\left(b x^{2}+(a+1) x y+a b y^{2}\right) x}{\left(x^{2}+2 b y x+y^{2}\right)^{2}} .
\end{aligned}
$$

Notice that if $b=0$ and $a=-1$ then $k \equiv-1$ so we exclude this case in the remainder of the discussion. Note also that the denominator $x^{2}+2 b x y+y^{2} \neq 0$ since $b^{2}<1$. Critical points are given by the pair of lines $b x^{2}+(a+1) x y+a b y^{2}=0$, except that the origin must be excluded, or else the plane on which the sectional curvature is being evaluated degenerates to a line. Furthermore, if $a>0$ the discriminant $(a+1)^{2}-$ $4 a b^{2}>(a+1)^{2}-4 a=(a-1)^{2}>0$ whereas if $a<0$ then $(a+1)^{2}-4 a b^{2} \geq(a+1)^{2} \geq 0$ : in fact $(a+1)^{2}-4 a b^{2}>0$ since we are excluding the case $b=0$ and $a=-1$.

Using the first derivative test it follows that

$$
2 a b y=\left(-(a+1) \pm \sqrt{(a+1)^{2}-4 a b^{2}}\right) x
$$

give local extrema, a local minimum for $2 a b y=(-(a+1)+$ $\left.\sqrt{(a+1)^{2}-4 a b^{2}}\right) x$, and local maximum for $2 a b y=(-(a+$ $\left.1)-\sqrt{(a+1)^{2}-4 a b^{2}}\right) x$. After much algebra, this minimum and maximum turn out to be given simply by $\left(a^{2} b^{2}+2 a b^{2}-\right.$ $\left.2 a^{2}+b^{2}-2 \pm 2(a-1) \sqrt{(a+1)^{2}-4 a b^{2}}\right) / 4\left(1-b^{2}\right)$. In the case where $b=0,0<|a|<1$ the function $k$ has a minimum of -1 for $y=0$ and maximum of $-a^{2}$ for $x=0$. Recall also that $a \neq 0$ or else the algebra does not belong to the given family.
Let us compare the two possible maxima in the case, where $-1 \leq a<0$. Subtracting gives

$$
\begin{aligned}
& \frac{b^{2}-4 a+6 a b^{2}+a^{2} b^{2}-b^{4}-2 a b^{4}-a^{2} b^{4}}{4\left(1-b^{2}\right)^{2}} \\
& -\frac{a^{2} b^{2}+2 a b^{2}-2 a^{2}+b^{2}-2-2(a-1) \sqrt{(a+1)^{2}-4 a b^{2}}}{4\left(1-b^{2}\right)} \\
& =\frac{2(a-1)\left(a-1+\sqrt{(a+1)^{2}-4 a b^{2}}\right)}{4\left(1-b^{2}\right)} .
\end{aligned}
$$

Considering the numerator above clearly $(a-1) \leq 0$; however, $a-1+\sqrt{(a+1)^{2}-4 a b^{2}}<0$ since $(a+1)^{2}-4 a b^{2}-$ $(1-a)^{2}=4 a\left(1-b^{2}\right)<0$ when $a<0$. Hence, when $a<0$ the maximum actually is $\left(b^{2}-4 a+6 a b^{2}+a^{2} b^{2}-b^{4}-2 a b^{4}-\right.$ $\left.a^{2} b^{4}\right) / 4\left(1-b^{2}\right)^{2}$.

We can summarize the maxima and minima for algebras 3.3-3.5 as follows:

(i) $a=1$ and consequently $b=0$ and sectional curvature is identically -1 .

(ii) $0<a<1,-1<b<1$ : maximum $\left(a^{2} b^{2}+\right.$ $\left.2 a b^{2}-2 a^{2}+b^{2}-2-2(a-1) \sqrt{(a+1)^{2}-4 a b^{2}}\right) / 4(1-$ $\left.b^{2}\right)$, minimum $\left(a^{2} b^{2}+2 a b^{2}-2 a^{2}+b^{2}-2+2(a-\right.$ 1) $\sqrt{\left.(a+1)^{2}-4 a b^{2}\right)} / 4\left(1-b^{2}\right)$.

(iii) $-1 \leq a<0,-1<b<1$ : minimum $\left(a^{2} b^{2}+2 a b^{2}-2 a^{2}+\right.$ $\left.b^{2}-2+2(a-1) \sqrt{(a+1)^{2}-4 a b^{2}}\right) / 4\left(1-b^{2}\right)$, maximum $=\left(b^{2}-4 a+6 a b^{2}+a^{2} b^{2}-b^{4}-2 a b^{4}-a^{2} b^{4}\right) / 4\left(1-b^{2}\right)^{2}$.

4.3.2. Ricci Curvature. Ricci as a type $(1,1)$ tensor which depends on $a$ and $b$ is given by

$$
\left[\begin{array}{ccc}
\frac{b^{2} a^{2}-2 a+2 b^{2} a-2+b^{2}}{2\left(1-b^{2}\right)} & \frac{a b(a-1)}{1-b^{2}} & 0 \\
-\frac{(a-1) b}{1-b^{2}} & \frac{b^{2} a^{2}+2 b^{2} a-2 a^{2}+b^{2}-2 a}{2\left(1-b^{2}\right)} & 0 \\
0 & 0 & \frac{b^{2} a^{2}+2 b^{2} a+b^{2}-2-2 a^{2}}{2\left(1-b^{2}\right)}
\end{array}\right]
$$

The Ricci eigenvalues are $\left((a+1)^{2} b^{2}-2\left(a^{2}+1\right)\right) / 2\left(1-b^{2}\right)$, $\left((a+1)^{2}\left(1-b^{2}\right) \pm \sqrt{\left(a^{2}-4 a b^{2}+2 a+1\right)(a-1)^{2}}\right) / 2\left(1-b^{2}\right)$. The first eigenvalue is always negative since $2\left(a^{2}+1\right)-(a+1)^{2} b^{2}>$ $(a-1)^{2}>0$. The second and third eigenvalues are real since $(a+1)^{2}-4 a b^{2}>b^{2}(a+1)^{2}-4 a b^{2}=b^{2}(a-1)^{2} \geq 0$. Moreover, taking the plus sign the second eigenvalue is clearly positive except in the very special case $b=0, a=-1$.
As regards the third eigenvalue it is negative, zero, or positive according as $(a+1)^{4} b^{4}-2\left(a^{4}+2 a^{3}+10 a^{2}+2 a+\right.$ $1) b^{2}+4 a(a+1)^{2}$ is negative, zero, or positive.

We have to treat $a=-1$ as a special case for which we obtain a negative or zero eigenvalue according as $b$ is nonzero or zero and we shall have to assume also that $b^{2}<1$.

Assuming then that $a \neq-1$, in order to have a nonnegative eigenvalue we need 


$$
b^{2} \geq \frac{a^{4}+2 a^{3}+10 a^{2}+2 a+1+\sqrt{\left(a^{4}+2 a^{3}+10 a^{2}+2 a+1\right)^{2}-4 a(a+1)^{6}}}{(a+1)^{4}}
$$

or

$$
b^{2} \leq \frac{a^{4}+2 a^{3}+10 a^{2}+2 a+1-\sqrt{\left(a^{4}+2 a^{3}+10 a^{2}+2 a+1\right)^{2}-4 a(a+1)^{6}}}{(a+1)^{4}}
$$

In the first case we find that

$$
\begin{aligned}
b^{2} & \geq \frac{a^{4}+2 a^{3}+10 a^{2}+2 a+1+\left(a^{2}-1\right)^{2}}{(a+1)^{4}} \\
& =\frac{2\left(a^{4}+a^{3}+4 a^{2}+a+1\right)}{(a+1)^{4}} .
\end{aligned}
$$

Now $2\left(a^{4}+a^{3}+4 a^{2}+a+1\right)-(a+1)^{4}=\left(a^{2}+1\right)(a-1)^{2}$ which is always nonnegative and positive if $a \neq 1$ which would give $b^{2} \geq 1$ contrary to hypothesis.

In the second case we find that

$b^{2}$

$$
\leq \frac{a^{4}+2 a^{3}+10 a^{2}+2 a+1-(a-1)^{2} \sqrt{(a+1)^{4}+4 a^{2}}}{(a+1)^{4}}
$$

or

$$
b^{2} \leq \frac{(a+1)^{4}-\left(2 a+\sqrt{(a+1)^{4}+4 a^{2}}\right)(a-1)^{2}}{(a+1)^{4}} .
$$

Now

$$
2 a+\sqrt{(a+1)^{4}+4 a^{2}} \geq 0
$$

with equality iff $a=-1$ and so if $b^{2}$ satisfies the first equality then necessarily $b^{2}<1$. Notice also that in order to have $b^{2} \geq$ 0 we must have that $0<a<1$.

Similarly, in order to have a nonpositive third eigenvalue

$$
b^{2} \leq \frac{(a+1)^{4}+\left(\sqrt{(a+1)^{4}+4 a^{2}}-2 a\right)(a-1)^{2}}{(a+1)^{4}}
$$

or

$$
b^{2} \geq \frac{(a+1)^{4}-\left(2 a+\sqrt{(a+1)^{4}+4 a^{2}}\right)(a-1)^{2}}{(a+1)^{4}} .
$$

However, since

$$
\sqrt{(a+1)^{4}+4 a^{2}}-2 a \geq 0
$$

it follows that

$$
\frac{(a+1)^{4}+\left(\sqrt{(a+1)^{4}+4 a^{2}}-2 a\right)(a-1)^{2}}{(a+1)^{4}} \geq 1 .
$$

Hence, if $b^{2}<1$ the first inequality is identically satisfied. To summarize,

(i) the third eigenvalue is positive if $b^{2}<\left((a+1)^{4}-(2 a+\right.$ $\left.\left.\sqrt{(a+1)^{4}+4 a^{2}}\right)(a-1)^{2}\right) /(a+1)^{4}$ and $0<a<1$,

(ii) the third eigenvalue is negative if $b^{2}>\left((a+1)^{4}-(2 a+\right.$ $\left.\left.\sqrt{(a+1)^{4}+4 a^{2}}\right)(a-1)^{2}\right) /(a+1)^{4}$ and $a \neq 0,-1$,

(iii) the third eigenvalue is zero if $b^{2}=\left((a+1)^{4}-(2 a+\right.$ $\left.\left.\sqrt{(a+1)^{4}+4 a^{2}}\right)(a-1)^{2}\right) /(a+1)^{4}$ and $0<a<1$ and $b \neq 0$

(iv) if $a=-1$ the third eigenvalue is negative unless $b=$ 0 in which case the second and third eigenvalues are zero.

The scalar curvature is $-\left(3\left(1-b^{2}\right)(a+1)^{2}+(a-1)^{2}\right) / 2(1-$ $\left.b^{2}\right)<0$. In the special case $a=-1$, which is the Lie algebra of $E(1,1)$, the group of motions of the pseudo-Euclidean plane, the eigenvalues simplify to $-2 /\left(1-b^{2}\right), \pm 2 b /\left(1-b^{2}\right)$ so we have either one positive and two negatives or one negative and two zeros if $b=0$.

4.4. Algebra $3.6(a=0), 3.7(a \neq 0)$. Nonzero brackets: $\left[e_{1}, e_{3}\right]=a e_{1}-e_{2},\left[e_{2}, e_{3}\right]=e_{1}+a e_{2}$.

$$
S=\left[\begin{array}{ccc}
e^{a z} \cos z & e^{a z} \sin z & x \\
-e^{a z} \sin z & e^{a z} \cos z & y \\
0 & 0 & 1
\end{array}\right] .
$$

Left-invariant vector fields: $e^{a z}\left(\cos z D_{x}-\sin z D_{y}\right)$, $e^{a z}\left(\sin z D_{x}+\cos z D_{y}\right),-D_{z}$.

Left-invariant one-forms: $e^{-a z}(\cos z d x-\sin z d y)$, $e^{-a z}(\sin z d x+\cos z d y),-d z$.

Right-invariant vector fields: $D_{x}, D_{y}, D_{z}+(a x+y) D_{x}+$ $(a y-x) D_{y}$. 
Right-invariant one-forms: $d x-(a x+y) d z, d y-(a y-$ $x) d z, d z$.

The space of derivations is given by $\left[\begin{array}{ccc}\alpha & \beta & \gamma \\ -\beta & \alpha & \delta \\ 0 & 0 & 0\end{array}\right]$ and the metric can be reduced to $\left[\begin{array}{lll}b & 0 & 0 \\ 0 & 1 & 0 \\ 0 & 0 & 1\end{array}\right]$ and again we have used scaling to simplify the metric. For the Lie algebra itself, it may be assumed without loss of generality that $a \geq 0$ and for the metric that $0<b \leq 1$ but not both of these conditions may hold simultaneously so we shall assume that the latter holds.

4.4.1. Sectional Curvature. The generic sectional curvature function $K(x, y)$ is given by

$$
K=\frac{\left(1+2 b-4 a^{2} b-3 b^{2}\right) x^{2}+8 a b(1-b) x y-\left(4 a^{2} b^{2}-b^{3}-2 b^{2}+3 b\right) y^{2}-4 a^{2} b^{2}+b^{3}-2 b^{2}+b}{4 b\left(b+x^{2}+b y^{2}\right)}
$$

We shall assume to begin with that $a(b-1) \neq 0$. The critical points of $K$ come from solving

$$
\begin{aligned}
& a y x^{2}-a y b-a y b x^{2}-b^{2} x y^{2}+a y b^{2}-a y^{3} b+x y^{2} \\
& \quad+a y^{3} b^{2}+b x-x b^{2}=0, \\
& b x a-b^{2} x a-a x b y^{2}+b^{2} x a y^{2}-b x^{3} a-y b^{2} x^{2}+y b \\
& \quad+y x^{2}-y b^{2}+a x^{3}=0 .
\end{aligned}
$$

Now $x$ times $(44)-y$ times $(45)$ gives $(b-1) b\left(2 a y x-x^{2}+y^{2}\right)=$ 0 . We shall discuss $b=1$ and $b \neq 0$ later so that $x^{2}=2 a y x+y^{2}$.
Substituting in (45) gives $-(-1+b)(y+a x)\left(2 a y x+b y^{2}+y^{2}+b\right)$ so that either $y+a x=0$ or $2 a y x+b y^{2}+y^{2}+b=0$. The first of these with $x^{2}=2 a y x+y^{2}$ gives $x=y=0$. The second gives $x^{2}+b\left(y^{2}+1\right)=0$ which would give $b \leq 0$ which is impossible. Thus, $y=-a x$. Substituting in the first gives $-b x\left(a^{2}+1\right)\left(a^{2} x^{2}+1\right)(-1+b)=0$. The only possibility is $x=$ $y=0$. The Hessian at the origin is $-4(1-b)^{2}\left(a^{2}+1\right) / b^{2}<0$ and so the origin gives a saddle point.

In the exceptional case we find that

$$
\begin{aligned}
& k=\frac{\left(b^{3}+2 b^{2}-4 a^{2} b^{2}-3 b\right) x^{2}+8 a b(1-b) x y+\left(2 b-4 a^{2} b-3 b^{2}+1\right) y^{2}}{4 b\left(b x^{2}+y^{2}\right)}, \\
& k_{x}=\frac{2(b-1) y\left(b x y+a b x^{2}-a y^{2}+x y\right)}{\left(b x^{2}+y^{2}\right)^{2}}, \\
& k_{y}=-\frac{2(b-1) x\left(b x y+a b x^{2}-a y^{2}+x y\right)}{\left(b x^{2}+y^{2}\right)^{2}}
\end{aligned}
$$

Since we are excluding $b=1$ and $a b \neq 0$ we deduce that the critical points come from solving $a b x^{2}+(b+1) x y-$ $a y^{2}=0$. Note that $b^{2}+2 b+1+4 a^{2} b=(b-1)^{2}+$ $4 b\left(1+a^{2}\right)>0$ so that there always two lines of critical points. Using the first derivative test it follows that $y=((b+$ $\left.\left.1-\sqrt{b^{2}+2 b+1+4 a^{2} b}\right) / 2 a\right) x$ gives a local, hence global and minimum, and $y=\left(\left(b+1+\sqrt{b^{2}+2 b+1+4 a^{2} b}\right) / 2 a\right) x$ gives a local, hence global and maximum. After much algebra, this maximum and minimum turn out to be given simply by $-(1 / 4 b)\left((b-1)^{2}+4 a^{2} b \pm 2(b-1) \sqrt{\left.(b+1)^{2}+4 a^{2} b\right)}\right.$.

Finally, if $b=1$ and $a \neq 0$ the space is of constant curvature $-a^{2}$. If $a=0$ but $b$ is not necessarily 1 , the maximum and minimum values just found remain valid: that is, $(b-1)(b+3) / 4 b$ and $(1-b)(3 b+1) / 4 b$.
4.4.2. Ricci Curvature. Ricci as a type $(1,1)$ tensor is

$$
\left[\begin{array}{ccc}
\frac{b^{2}-1-4 a^{2} b}{2 b} & \frac{a(1-b)}{b} & 0 \\
a(1-b) & \frac{1-4 a^{2} b-b^{2}}{2 b} & 0 \\
0 & 0 & -\frac{4 b a^{2}+1+b^{2}-2 b}{2 b}
\end{array}\right]
$$

and the eigenvalues are $-\left((b-1)^{2}+4 a^{2} b\right) / 2 b,\left(-4 a^{2} b \pm(1-\right.$ b) $\left.\sqrt{\left(b^{2}+4 b a^{2}+2 b+1\right)}\right) / 2 b$. The scalar curvature is $-((b-$ $\left.1)^{2}+12 a^{2} b\right) / 2 b$ which is negative. Two of the eigenvalues are unconditionally negative but the third eigenvalue can be positive, zero, or negative. 
If the third eigenvalue is zero then $a^{2}=\left((1-b)^{2} \pm(1-\right.$ b) $\left.\sqrt{(1-b)^{2}+4(1+b)^{2}}\right) / 4 b$. However, if we take the minus sign then $a^{2}<0$, so we must take the plus sign and, in so doing, we obtain precisely the condition to have a zero eigenvalue. Indeed rearranging the quadratic condition gives that the third eigenvalue $\geq 0$ provided that $a^{2} \leq\left(\left(1-b^{2}\right)+\right.$ $\left.(1-b) \sqrt{5 b^{2}+6 b+5}\right) / 8 b$ and $\leq 0$ provided that $a^{2} \geq((1-$ $\left.\left.b^{2}\right)+(1-b) \sqrt{5 b^{2}+6 b+5}\right) / 8 b$.

4.5. Algebra $3.8 \mathfrak{g l}(2, \mathbb{R})$. Nonzero brackets: $\left[e_{1}, e_{3}\right]=-2 e_{2}$, $\left[e_{1}, e_{2}\right]=e_{1},\left[e_{2}, e_{3}\right]=e_{3}$.

$$
S=\left[\begin{array}{ccc}
e^{-y}+2 x z+x^{2} z^{2} e^{y} & x+x^{2} z e^{y} & -x^{2} e^{y} \\
2 z\left(1+x z e^{y}\right) & 1+2 x z e^{y} & -2 x e^{y} \\
-z^{2} e^{y} & -z e^{y} & e^{y}
\end{array}\right] .
$$

Left-invariant vector fields: $e^{-y} D_{x}+2 z D_{y}-z^{2} D_{z}, D_{y}-$ $z D_{z}, D_{z}$.

Left-invariant one-forms: $e^{y} d x,-2 z e^{y} d x+d y, d z-$ $z^{2} e^{y} d x+z d y$.

Right-invariant vector fields: $D_{x}, D_{y}-x D_{x}, e^{-y} D_{z}-$ $x^{2} D_{x}+2 x D_{y}$.

Right-invariant one-forms: $d x+x d y-x^{2} e^{y} d z, d y-$ $2 x e^{y} d z, e^{y} d z$.

We note that $\mathfrak{g} \mathfrak{I}(2, \mathbb{R})$ is isomorphic to $\mathfrak{g} \mathfrak{p}(2,1)$ and we rewrite the algebra as $\left[e_{1}, e_{2}\right]=-e_{3},\left[e_{2}, e_{3}\right]=e_{1},\left[e_{3}, e_{1}\right]=e_{2}$. We take the Lorentz inner product on $\mathbb{R}^{3}$ as $\left[\begin{array}{ccc}1 & 0 & 0 \\ 0 & 1 & 0 \\ 0 & 0 & -1\end{array}\right]$ so that the group $S O(2,1)$ acts on its Lie algebra as automorphisms.
A symmetric $3 \times 3$ matrix $A$ can be diagonalized; that is, there exists $P \in S O(2,1)$ such that $P^{t} A P$ is $\left[\begin{array}{lll}a & 0 & 0 \\ 0 & b & 0 \\ 0 & 0 & c\end{array}\right]$.

4.5.1. Sectional Curvature. The generic sectional curvature function is given by

$$
\begin{gathered}
K(x, y)=\frac{1}{4\left(a b+b c x^{2}+a c y^{2}\right) a b c}\left(a^{3} b-2 a^{2} b^{2}+a b^{3}\right. \\
-3 a b c^{2}-2 a^{2} b c-2 a b^{2} c \\
+\left(2 b^{2} c^{2}+b c^{3}-3 a^{2} b c+b^{3} c+2 a b^{2} c-2 a b c^{2}\right) x^{2} \\
\left.+\left(a^{3} c+2 a^{2} c^{2}+2 a^{2} b c-2 a b c^{2}-3 a b^{2} c+a c^{3}\right) y^{2}\right), \\
K_{x} \\
=\frac{2 x\left(c(b-a)(a+b+c) y^{2}+b(a+c)(b-a+c)\right)}{\left(b c x^{2}+a c y^{2}+a b\right)^{2}}, \\
K_{y} \quad \\
=\frac{2 y\left(c(a-b)(a+b+c) x^{2}+a(b+c)(a-b+c)\right)}{\left(b c x^{2}+a c y^{2}+a b\right)^{2}} .
\end{gathered}
$$

Clearly $x=y=0$ gives a critical point and critical value of $\left((a-b)^{2}-c(2 a+2 b+3 c)\right) / 4 a b c$. In order to have a second solution for which $x y \neq 0$ we require that $(a-b-c)(a-$ $b)<0,(a-b+c)(a-b)<0$ : however, these conditions are incompatible.

If $c=a \neq b$ then the conditions to obtain a critical point become $x^{2}=(b-2 a)(a+b) /(a-b)(2 a+b), y^{2}=2 b^{2} /(a-$ $b)(2 a+b)$ and similarly if $a=b \neq c$ and a fortiori if $a=b=c$.

In the nongeneric case

$$
\begin{aligned}
k(x, y) & =\frac{\left(a^{3}+2 a^{2} c-3 a b^{2}+a c^{2}+2 a^{2} b-2 a b c\right) x^{2}+\left(-3 a^{2} b+b^{3}+2 b^{2} c+b c^{2}+2 a b^{2}-2 a b c\right) y^{2}}{\left(a x^{2}+b y^{2}\right) a b c}, \\
k_{x} & =\frac{2 x y^{2}(a-b)(a+b+c)}{c\left(a x^{2}+b y^{2}\right)^{2}} \\
k_{y} & =-\frac{2 x^{2} y(a-b)(a+b+c)}{c\left(a x^{2}+b y^{2}\right)^{2}}
\end{aligned}
$$

The critical points are of the form $(x, 0)$, where $x \neq 0$ and $(0, y)$ where $y \neq 0$ giving, respectively, critical values of $((a+$ $\left.c)^{2}+b(2 a-3 b-2 c)\right) / 4 a b c$ and $\left((b+c)^{2}-a(3 a-2 b+2 c)\right) / 4 a b c$.

Suppose that $a=b \neq c$. Then in the nongeneric case $K \equiv$ $c / 4 a^{2}$. If $a=c \neq b$ we find that the numerators of $k_{x}$ and $k_{y}$ both contain a factor of $2 a^{2}-a b-b^{2}=(2 a+b)(a-b)$, which is not zero and so we do not get any more critical points.

To summarize, the maximum and minimum sectional curvatures are to be found among $\left((a-b)^{2}+c(2 a+2 b-\right.$ $3 c)) / 4 a b c,\left((a+c)^{2}+b(2 a-3 b-2 c)\right) / 4 a b c$, and $\left((b+c)^{2}-\right.$ $a(3 a-2 b+2 c)) / 4 a b c$.
4.5.2. Ricci Curvature. In this case Ricci as a type $(1,1)$ tensor is $(1 / 2 a b c)\left[\begin{array}{ccc}a^{2}-(b+c)^{2} & 0 & 0 \\ 0 & b^{2}-(a+c)^{2} & 0 \\ 0 & 0 & c^{2}-(a-b)^{2}\end{array}\right]$ and the scalar curvature is $-\left((a-b)^{2}+c(2 a+2 b+c)\right) / 2 a b c$. It is impossible for Ricci to be zero or negative definite: $b+c>a, a+c>b, a-b>c$ are mutually incompatible and hence the metric can never be of constant curvature. Furthermore, if one eigenvalue is zero, two of them are zero and the third is negative.

4.6. Algebra $3.9 \mathfrak{g} \mathfrak{p}(3)$. Nonzero brackets: $\left[e_{1}, e_{2}\right]=e_{3}$, $\left[e_{2}, e_{3}\right]=e_{1},\left[e_{3}, e_{1}\right]=e_{2}$. 


$$
S=\left[\begin{array}{ccc}
\cos x \cos y \cos z-\sin x \sin z & \sin x \cos y \cos z+\cos x \sin z & -\sin y \cos z \\
-\cos x \cos y \sin z-\sin x \cos z & -\sin x \sin z \cos y+\cos x \cos z & \sin y \sin z \\
\cos x \sin y & \sin x \sin y & \cos y
\end{array}\right] .
$$

Left-invariant vector fields: $D_{x}, \cos x D_{y} \quad-$ $(\sin x \cos y / \sin y) D_{x}+(\sin x / \sin y) D_{z}, \sin x D_{y}+$ $(\cos x \cos y / \sin y) D_{x}-(\cos x / \sin y) D_{z}$.

Left-invariant one-forms: $d x+\cos y d z, \cos x d y+$ $\sin x \sin y d z,-\sin x d y+\cos x \sin y d z$.

Right-invariant vector fields: $D_{z},(\sin z / \sin y) D_{x}+$ $\cos z D_{y}-(\cos y \sin z / \sin y) D_{z},(\cos z / \sin y) D_{x}-$ $\sin z D_{y}-(\cos y \cos z / \sin y) D_{z}$.

Right-invariant one-forms: $d z+\cos y d x$, $\sin y \sin z d x+\cos z d y, \sin y \cos z d x-\sin z d y$.

We can diagonalize the metric using an orthogonal transformation so that its matrix is $\left[\begin{array}{lll}a & 0 & 0 \\ 0 & b & 0 \\ 0 & 0 & c\end{array}\right]$. If $a=b=c$ the space is of constant curvature $1 / 4 a$.

4.6.1. Sectional Curvature. We proceed as before and find the sectional curvature of a tangent 2-plane that is transverse to the $z$-axis for which a basis consists of $\left\{X=D_{x}+x D_{z}, Y=\right.$ $\left.D_{y}+y D_{z}\right\}$. Thus,

$$
\begin{aligned}
& K(x, y)=\frac{1}{4\left(a b+a c y^{2}+b c x^{2}\right) a b c}\left(a^{3} b-2 a^{2} b^{2}\right. \\
& +a b^{3}-3 a b c^{2}+2 a^{2} b c+2 a b^{2} c \\
& +\left(-2 c^{2} b^{2}+c^{3} b-3 a^{2} b c+c b^{3}+2 a b^{2} c+2 a b c^{2}\right) \\
& \quad \cdot x^{2} \\
& +\left(a^{3} c-2 a^{2} c^{2}+2 a^{2} b c+2 a b c^{2}-3 a b^{2} c+a c^{3}\right) \\
& \left.\quad \cdot y^{2}\right) .
\end{aligned}
$$

The partial derivatives are given by

$K_{x}$

$$
=\frac{2 x\left(b(c-a)(a+c-b)+c(b-a)(a-c+b) y^{2}\right)}{\left(a b+a c y^{2}+c x^{2} b\right)^{2}},
$$

$K_{y}$

$=\frac{2 y\left(a(b-c)(a-b-c)+c(a-b)(a-c+b) x^{2}\right)}{\left(a b+a c y^{2}+c x^{2} b\right)^{2}}$.

In order to have a solution other than $x=y=0$ we need

$b(a-c)(a-b+c) / c(a-b)(a+b-c)<0, a(b-c)(a-b-c) / c(a-$ $b)(a+b-c)<0$ hence $(a-c)(a-b+c) /(a-b)(a+b-c)<0$, $(b-c)(a-b-c) /(a-b)(a+b-c)<0$.
Then $a, b, c$ must be distinct. Note that the inequalities are invariant under interchange of $a$ and $b$. Hence, it is sufficient to check the cases: $a<b<c, c<a<b, a<c<b$. Thus, the first inequality gives $a+b-c<0$ whereas the second $a+b-c>$ 0 . If $c<a<b$ the second inequality gives $a>b+c$ which contradicts $a<b$. Finally if $a<c<b$ the first inequality gives $b>a+c$ which contradicts $a<b$. Thus, $(0,0)$ is the only critical point and $K(0,0)=\left((a-b)^{2}+c(2 a+2 b-3 c)\right) / 4 a b c$.

We can find all sectional curvatures by permuting the roles of $x, y$, and $z$. The maximum and minimum values will be found among

$$
\begin{aligned}
& R_{1212}=\frac{(a-b)^{2}+c(2 a+2 b-3 c)}{4 a b c}, \\
& R_{2323}=\frac{(b-c)^{2}+a(2 b+2 c-3 a)}{4 a b c}, \\
& R_{3131}=\frac{(c-a)^{2}+b(2 c+2 a-3 b)}{4 a b c} .
\end{aligned}
$$

4.6.2. Ricci Curvature. Ricci is given by (1/ $2 a b c)\left[\begin{array}{ccc}a^{2}-(b-c)^{2} & 0 & 0 \\ 0 & b^{2}-(a-c)^{2} & 0 \\ 0 & 0 & c^{2}-(a-b)^{2}\end{array}\right]$. Three eigenvalues can be positive but only if they are all equal and $a=b=c$; otherwise, we can have two zero and one positive or one positive and two negative eigenvalues, respectively. The metric is of constant curvature $1 / 4 a$ only in the case $a=b=c$.

The scalar curvature is given by $\left(2 a b+2 b c+2 c a-a^{2}-b^{2}-\right.$ $\left.c^{2}\right) / 2 a b c$. Depending on the values of $a, b, c$ it can be positive, zero, or negative. For example, if $c=(\sqrt{a} \pm \sqrt{b})^{2}$ it is zero.

4.7. Dimension 3: Decomposable. Nonzero brackets: $\left[e_{1}, e_{2}\right]=$ $e_{2}$.

$$
S=\left[\begin{array}{cc}
e^{x+z} & y e^{z} \\
0 & e^{z}
\end{array}\right]
$$

Left-invariant vector fields: $D_{x}, e^{x} D_{y}, D_{z}$

Left-invariant one-forms: $d x, e^{-x} d y, d z$.

Right-invariant vector fields: $-\left(D_{x}+y D_{y}\right), D_{y}, D_{z}$.

Right-invariant one-forms: $-d x, d y-y d x, d z$.

The space of derivations is given by $\left[\begin{array}{lll}\alpha & 0 & 0 \\ \delta & \beta & 0 \\ \epsilon & 0 & \gamma\end{array}\right]$ and the metric can be reduced to $\left[\begin{array}{ccc}1 & 0 & 0 \\ 0 & 1 & 2 a \\ 0 & 2 a & 1\end{array}\right]$, where $|a|<1 / 2$. 
4.7.1. Sectional Curvature. The generic sectional curvature function is given by

$$
\begin{aligned}
& K(x, y) \\
& \quad=\frac{1-5 a^{2}-4 a^{3} y-a^{2} y^{2}+4 a^{4} x^{2}-a^{2} x^{2}}{(2 a-1)(2 a+1)\left(1+4 a y+y^{2}+x^{2}-4 a^{2} x^{2}\right)}, \\
& K_{x}=\frac{2 x(1-2 a)(2 a+1)}{\left(\left(4 a^{2}-1\right) x^{2}-1-4 a y-y^{2}\right)^{2}}, \\
& K_{y}=\frac{2(2 a+y)}{\left(\left(4 a^{2}-1\right) x^{2}-1-4 a y-y^{2}\right)^{2}} .
\end{aligned}
$$

The only critical point is $(0,-2 a)$ and the determinant of the Hessian is given by $4 /(1-2 a)^{3}(2 a+1)^{3}$ which is positive. Furthermore, $K_{x x}(0,-2 a)$ is given by $2 /(1-2 a)(2 a+1)>0$ and thus gives a local and in fact global minimum of (1 $\left.a^{2}\right) /\left(4 a^{2}-1\right)$.

In the nongeneric case we have that $k(x, y) \equiv a^{2} /\left(1-4 a^{2}\right)$. Hence, the sectional curvature varies between a minimum of $\left(a^{2}-1\right) /\left(1-4 a^{2}\right)$ and maximum of $a^{2} /\left(1-4 a^{2}\right)$ inclusive.

4.7.2. Ricci Curvature. The Ricci tensor as a type $(1,1)$ tensor is $\left[\begin{array}{ccc}\left(-1+2 a^{2}\right) /\left(1-4 a^{2}\right) & 0 & 0 \\ 0 & \left(-1+2 a^{2}\right) /\left(1-4 a^{2}\right) & 0 \\ 0 & 2 a /\left(1-4 a^{2}\right) & 2 a^{2} /\left(1-4 a^{2}\right)\end{array}\right]$ and gives Ricci eigenvalues of $\left(-1+2 a^{2}\right) /\left(1-4 a^{2}\right)$ of multiplicity two and $2 a^{2} /\left(1-4 a^{2}\right)$ of multiplicity one.

\section{Competing Interests}

The authors declare that they have no competing interests.

\section{References}

[1] J. Milnor, "Curvatures of left invariant metrics on Lie groups," Advances in Mathematics, vol. 21, no. 3, pp. 293-329, 1976.

[2] F. Hindeleh and G. Thompson, "Killing's equations for invariant metrics on Lie groups," Journal of Geometric Mechanics, vol. 3, no. 3, pp. 323-335, 2011.

[3] J. Patera, R. T. Sharp, P. Winternitz, and H. Zassenhaus, "Invariants of real low dimension Lie algebras," Journal of Mathematical Physics, vol. 17, no. 6, pp. 986-994, 1976. 


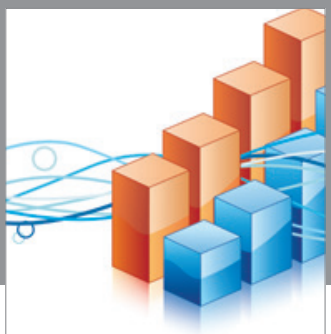

Advances in

Operations Research

vatem alat4

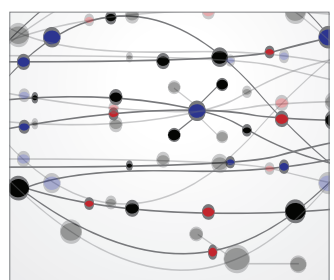

\section{The Scientific} World Journal
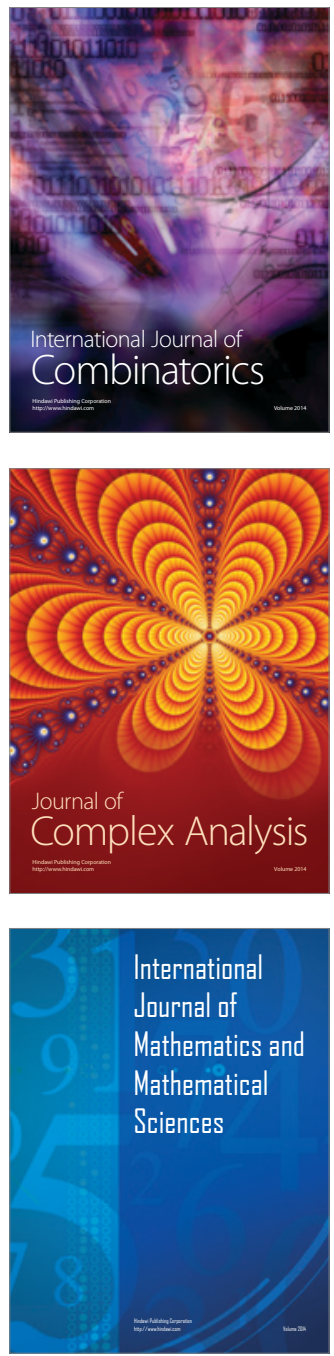
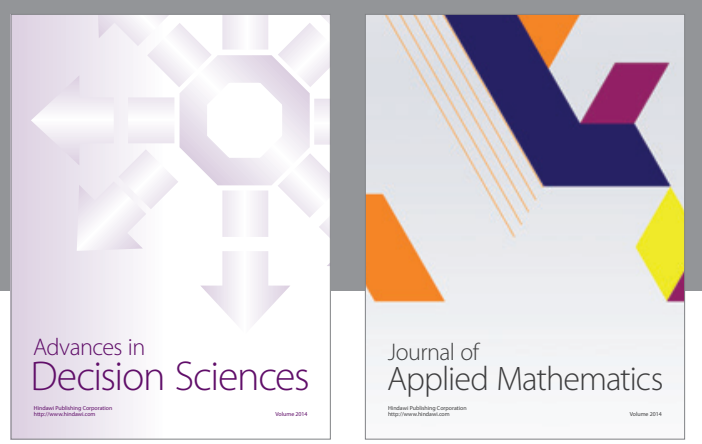

Algebra

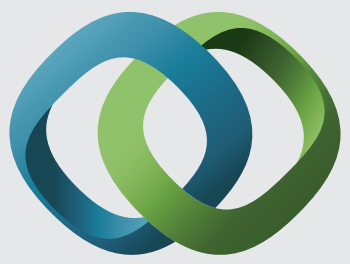

\section{Hindawi}

Submit your manuscripts at

http://www.hindawi.com
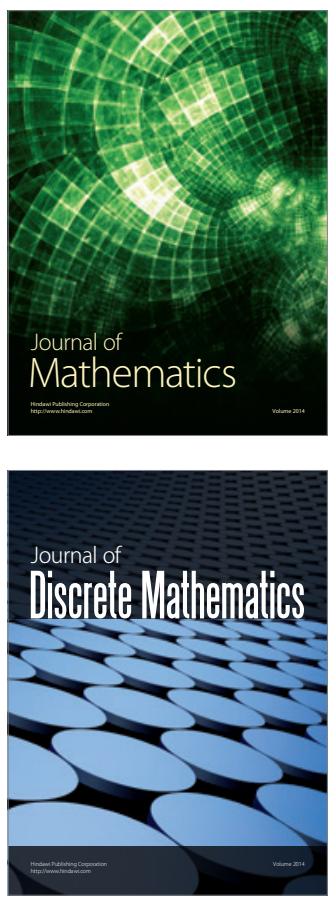

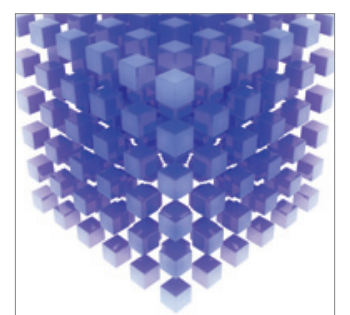

Mathematical Problems in Engineering
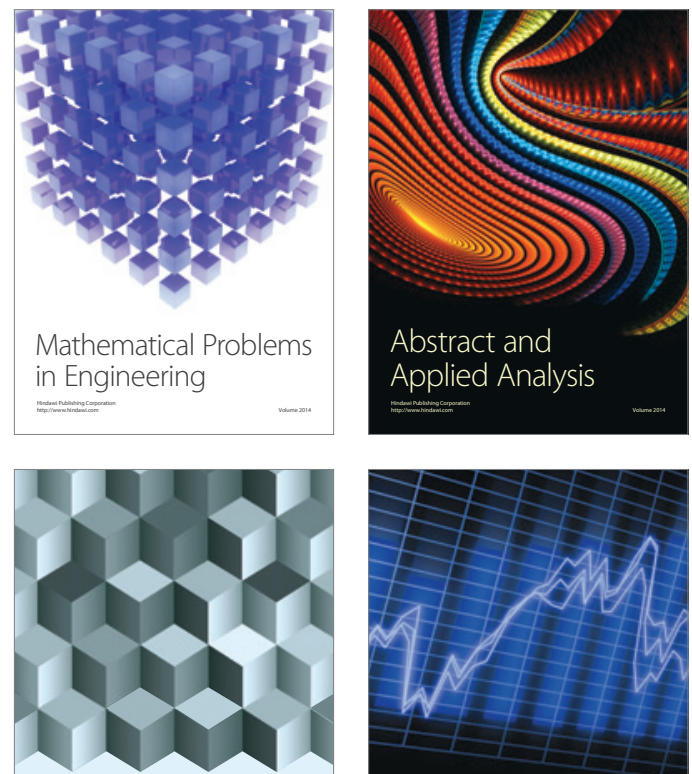

Journal of

Function Spaces

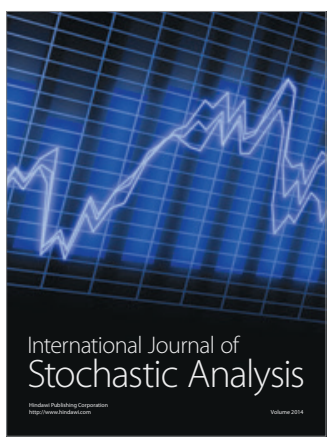

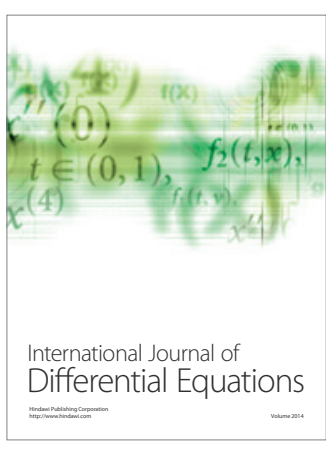
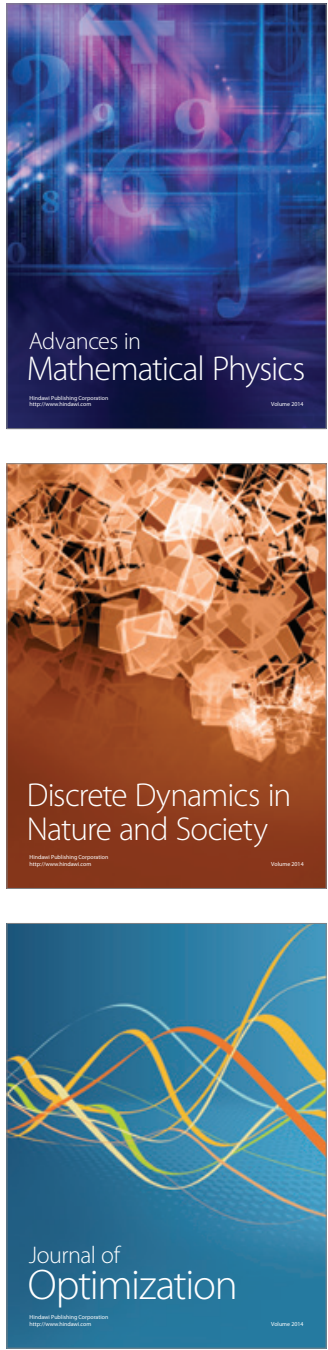